\title{
Cloning and Sequence Analysis of Gene Encoding OipA from Iranian Patients with Helicobacter pylori Infection
}

\author{
Mohaddese Mahboubi 1,*, Tahereh Falsafi 1, Majid Sadeghizadeh 2 \\ ${ }^{1}$ Department of Biology, Faculty of Basic Sciences, Alzahra University, Tehran, IR IRAN \\ ${ }^{2}$ Department of Genetics, Faculty of Biological Sciences, Tarbiat Modares University, Tehran, IR IRAN \\ ${ }^{*}$ Corresponding Author:Mohaddese Mahboubi, Department of Biology, Faculty of Basic Sciences, Alzahra University, Tehran, IR IRAN. Tel: +98- \\ 2188058912, Fax:+98-2188058912, E-mail: mahboubi@barijessence.com
}

Received: August 16,2014; Revised: September 20,2014; Accepted: October 07, 2014

Background: Outer inflammatory protein A (OipA) is one of the important adhesins of $H$. pylori and a valuable candidate for vaccine development. Its gene is under "on-off" switch status which correlates with OipA protein expression.

Objectives: We aimed to obtain a recombinant OipA clone (with "on" status) from an Iranian clinical isolate.

Materials and Methods: A clinical H. pylori-isolate demonstrating high expression for an outer membrane protein (OMP) with an apparent MW of 33-35 kDa was selected. oipA specific primer was designed according to oipA sequences from B8 strain. The purified PCR-product was sequenced and submitted to Gene Bank. The pET-28a plasmid and E. coli $\mathrm{DH}_{5} \alpha$ were used for cloning and transformation. The recombinant plasmid was transferred to E. coli BL21 (DE3). Extracted proteins were purified and presence of OipA was confirmed by western blotting using both anti His-tag monoclonal antibody and anti-OipA specific antibody.

Results: The sequence of the oipA gene and the MW of the purified recombinant OipA protein consisted on $924 \mathrm{bp}$ and 33-35 kDa, respectively. Its identity with other published oipA genes was $92-96 \%$; highest identity was observed with that of a Mexican oipA clone, obtained from a H. pylori strain associated with severe symptoms.

Conclusions: Recombinant oipA clone obtained in this work, may be a functional oipA gene with "on" status, associated with more severe outcomes of $H$. pylori infection.

Keywords: Cloning; Helicobacter pylori; Iran; OipA

\section{Background}

Helicobacter pylori is a human pathogen that infects half of the world's population and causes gastritis, gastric and duodenal ulcers, gastric cancer, and Mucosa Associated Lymphoid Tissue lymphoma. Adhesion of $H$. pylori to gastric epithelial cells may be the first important step in the pathogenesis of $H$. pylori infection (1). Together with CagA and VacA that are two well-described virulence factors of $H$. pylori, OipA (outer inflammatory protein A) is an important virulence factor which plays role in the special interaction with host cell membrane and favors production of a proinflammatory cytokine, IL-8 (2).

Numerous studies on OipA have demonstrated that the oip $A$ gene expression into a $33-35 \mathrm{kDa}$ protein is regulated by the slipped-strand repair mechanism that changes the number of CT dinucleotide repeats in the 5 '-region of the oipA gene. Thus, a switch "on" status is functional and switch "off” is nonfunctional $(3,4)$.
The oipA gene has been cloned from various regions of the world, including South America, North America, Europe, Asia, East Asia and Japan (4-6). However, molecular comparison of the recombinant clones has demonstrated some polymorphism in the variable regions of the oipA gene suggesting geographical location related differences among them. Furthermore, Torres-Morquecho et al., (2010) have found the significant positive selection acting on variable regions of oipA, that could be related to differences in its risk for gastroduodenal diseases among different human populations (6).

There is no study from Iran that has reported cloning the full gene of oipA. In a work performed by Teymournejad et al., in Iran (2013), not only a universal laboratory $H$. pylori strain (26695) have been used for molecular cloning, but also the sequences of their clone contained only $783 \mathrm{bp}$, and the related protein had $30 \mathrm{kDa}$ (7). Yamaoka et al., (2000), the leading 
Table 1. Specification of primers in PCR reaction

\begin{tabular}{lccc}
\hline Primers & \multicolumn{1}{c}{ Forward (5'-3') } & Reverse (5'-3') & Product size \\
\hline vacA $s$ & ATGGAAATACAACAAACACAC & CTGCTTGAATGCGCAAAC & 285 \\
vacAm & CAATCTGTCCAATCAAGCCAG & GCGTCTAAATAATTCCAAGG & 642 \\
ureC2 & AACGCCCTTTCTTCTCAAGC & TTCGCTCACAAACTTATCCC & 123 \\
ureC1 & TGATAAGCGCGAGCCACAAC & ACCGGAGCCACCTTATAAGC & 295 \\
cagA & ATGACTAACGAAACTATTGATC & TATCGCCAAGAGTGAATTTAG & 821 \\
\hline
\end{tabular}

investigator working in this field, has reported that functional OipA protein had $34 \mathrm{kDa}$ weight (4) and the full gene of oipA had almost $930 \mathrm{bp}$.

\section{Objectives}

Considering the important role of OipA in the pathogenesis of $H$. pylori infection and its variability among the strains isolated from various geographical regions, we aimed to obtain a recombinant OipA from a strain isolated from the Iranian patients. For selection of strain, we have taken into consideration two important factors: selection of strain from patients with $H$. pylori related symptoms, as well as OipA expression ability of the strain.

\section{Material and Methods}

\subsection{Isolation and Identification of H. pylori Strains}

To isolate the $H$. pylori strain, antral biopsies obtained from the patients suffering from the symptoms of $H$. pylori infection was processed according to the previously described protocol (4). The protocol was approved by the local Bioethics Committee. Written informed consent was obtained from all the patients prior to the participation in the study. In brief, the strains were routinely cultivated on Brucella agar (Biolife; Albimi, Italiana S. r. L. Viale Monza 27220128 Milano-Italia) supplemented with 5\% sheep blood, polymyxin-B (8 mg. $\left.\mathrm{l}^{-1}\right)$, amphotericin (2 mg.1$\left.{ }^{1}\right)$ and vancomycin $\left(6 \mathrm{mg} . \mathrm{l}^{-1}\right)$ and were incubated for 37 days under microaerophilic conditions $\left(10 \% \mathrm{CO}_{2}\right.$ and $95 \%$ humidity) at $37^{\circ} \mathrm{C}$. Identification of $\mathrm{H}$. pylori strains were performed by morphology of the colonies, Gram staining, biochemical tests (oxidase, rapid urease, and nitrate) and PCR amplification of $H$. pylori $16 \operatorname{SrRNA}$ and ureC.

\subsection{Selection of Strain for Cloning}

Clinical $H$. pylori strains were screened for the presence of a 33-35 $\mathrm{kDa}$ protein on SDS-PAGE pro- files of OMPs. OMPs were prepared according to the previously described method (8). A strain (S15), demonstrating high expression for a protein with an apparent MW of 33-35 kDa, was selected for cloning of the oip $A$ gene. For this strain, the vacA/cagA genotype/status was also determined according the previously determined PCR primers and PCR protocols (5, 9).

\subsection{Amplification of oipA Gene}

$H$. pylori DNA was obtained using the DNA extraction kit (SinaClone, Iran) according to the manufacturer's instructions. The primer sequence was designed according to the available genome sequence of oip $A$ from $H$. pylori B8 strain (Table 1). The oipA forward primer with $B a m \mathrm{HI}$ endonuclease site was 5'-CCGGATCCATGAAAAAAGCTCTCTTACT-3' and the oipA reverse primer with an $X h o$ I endonuclease site was $5^{\prime}$ CGCGGCTCGAGTTAATGTTTGTTTTTAAAGTT-3' . The PCR reaction was performed in $25 \mu 1$ mixture containing the DNA template (100 $\mathrm{ng}), 0.2 \mathrm{mM}$ of each dNTP, $0.4 \mu \mathrm{M}$ of each primer, $2 \mathrm{mM} \mathrm{MgCl}_{2}, 0.5$ U Taq DNA polymerase and PCR buffer (1x) (SinaClone, Iran). Following denaturation at $95^{\circ} \mathrm{C}(2$ $\mathrm{min})$, the fragment was amplified through 30 cycles as follows: $95^{\circ} \mathrm{C}(30 \mathrm{~s}), 65^{\circ} \mathrm{C}(45 \mathrm{~s}), 72^{\circ} \mathrm{C}(90 \mathrm{~s})$; the extension was continued at $72^{\circ} \mathrm{C}$ for $10 \mathrm{~min}$. Aliquots of the PCR product were electrophoresed in a $1 \%$ $(\mathrm{W} / \mathrm{V})$ agarose gel in $0.5 \times$ Tris-borate-EDTA buffer (90 mM Tris-HCl, $90 \mathrm{mM}$ boric acid, $2 \mathrm{mM}$ EDTA), and stained in DNA green safe (Sinaclone, Iran) at 4 ppm and predicted 924 bp was visualized under UV.

\subsection{Sequence Analysis}

Before cloning, the PCR product of the oipA gene was sequenced (Macrogen, Korea) and the results of sequencing were submitted to GenBank that were accepted under accession number KJ816695 (http://www.ncbi.nlm.nih.gov/nuccore/KJ816695). 
Sequence comparison between oip $A$ gene obtained in this work and those previously obtained in other parts of the world was also performed.

\subsection{Construction and Purification of Recombinant oipA}

To construct a recombinant plasmid containing the oipA gene, the PCR product was purified with the Ambiclean kit (Vivantis Technologies Sdn Bhd, Malaysia). The pET-28a containing His tag sequences and purified PCR product were both digested by two endonucleases including the BamHI and Xhol (Thermo Scientific, USA) to make cohesive ends. The resulting fragments of pET-28a and oipA were ligated by T4 DNA ligase (Thermo Scientific, USA). The pET-oipA construct was transformed into E. coli $\mathrm{DH}_{5} \alpha$ and was cultured on Luria Bertani (LB) Agar with Kanamycin (30 $\left.\mu \mathrm{g} . \mathrm{ml}^{-1}\right)$. The recombinant clones were isolated and the recombinant plasmid was extracted for further verification by PCR and restriction enzyme digestion . In order to obtain a better expression, the extracted recombinant plasmid construct was transformed into E. coli BL21 (DE3). Induction was performed in LB broth by $1 \mathrm{mM}$ IPTG (Isopropyl $\beta$-D-1-thiogalactopyranoside).

\subsection{Purification and Identification of Recombinant OipA Protein}

OipA protein was purified by Ni-NTA affinity chromatography (Novagen, USA) and its presence was confirmed by Western blotting using anti-His-tag monoclonal antibody. For this purpose, the purified protein was separated by $12 \%$ SDS-PAGE and was transferred onto a PVDF membrane. The membrane was blocked with blocking solution containing albumin $1 \%$ for $1 \mathrm{~h}$ at $25^{\circ} \mathrm{C}$. The blot was directly treated with 100 mU.ml-1 ${ }^{-1}$ Anti-His ${ }_{6}$ Peroxidase solution (Roche) and incubated for $1 \mathrm{~h}$ at $25^{\circ} \mathrm{C}$. The blot was washed with washing solution $(3 \times$, each $5 \mathrm{~min})$. Predicted desired band was detected by TMB $(3,3,5,5-$ Tetramethylbenzidine) as substrate.

\subsection{Production of Monospecific Polyclonal Antibodies} (pAbs) Against OipA and Specific Identification of Recombinant OipA

For this purpose, a white New Zealand rabbit (Razi institute, Iran), was immunized, subcutaneously by purified antigen (OipA) with Freund's adjuvant three times at 2 week intervals, and blood samples were taken two weeks after third injection. The resultant pAbs were purified using affinity chromatography
(Sepharose 4B coupled with H. pylori OMPs). For Western blotting, the SDS-PAGE containing proteins were transferred onto nitro-cellulose sheets using a semi-dry electrotransfer apparatus (Akhtarian Co, Iran). The filters were processed according to the protocol previously described (10).

\section{Results}

The selected clinical H. pylori strain (S15), which was isolated from a patient with severe gastritis expressed a protein with an apparent MW of 33-35 $\mathrm{kDa}$. Its vacA/cagA genotype/status determined by PCR was $s \operatorname{lm} 2 v a c A$ and $c a g A$ positive, respectively (Figure 1). The product of the PCR amplification for the oip-A gene was $924 \mathrm{bp}$. The result of restriction digestion of recombinant pET-28a plasmid confirmed the presence of the oip-A gene (Figure 2). The result of sub-cloning into $E$. coli $\mathrm{BL}$ (DE3), as well as its PCR product was similar to those of $E$. coli $\mathrm{DH} 5 \alpha$. The purified protein from the OipA expression vector $E$. coli BL (DE3) showed an apparent MW of $34 \mathrm{kDa}$ (Figure 3 A-B).

The purified OipA protein was detected by antiHis-tag monoclonal antibody in Western blotting and confirmed the presence of $34 \mathrm{kDa}$ protein (Figure 3C). Specific identification of recombinant OipA with anti $H$. pylori OipA by Western blotting was demonstrated in Figure 4.

The DNA sequences of the oipA gene from this

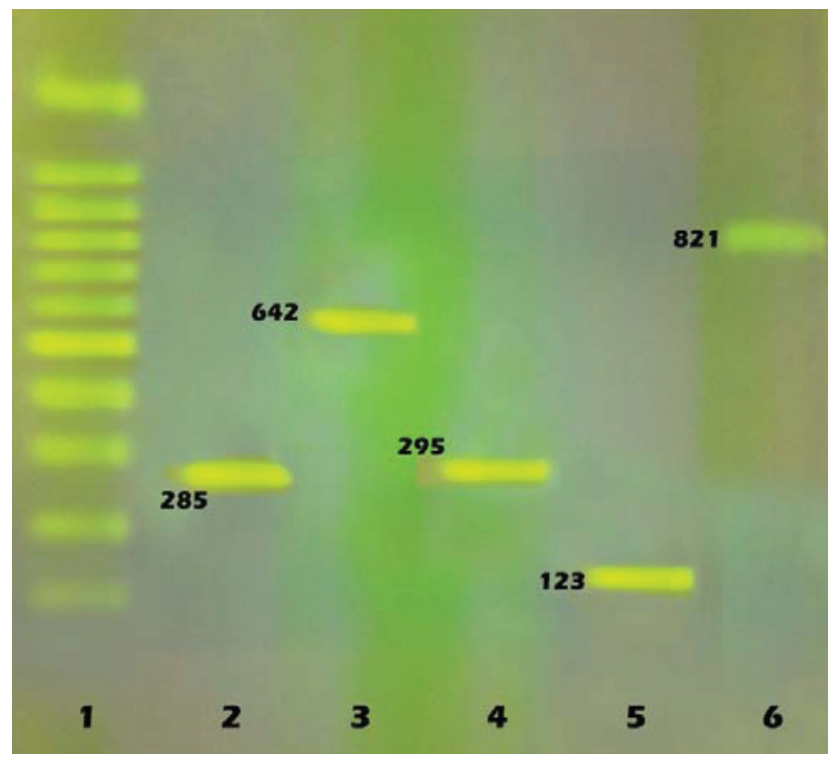

Figure 1. The results of PCR amplification $\operatorname{vacA}(s, m)$, cagA, and ureC. Lane 1: MW standard, Lane 2: vacAs1, lane 3: vacAm2, lane 4: ureC1, lane 5: ureC2, and lane 6: cagA according to the Table 1 


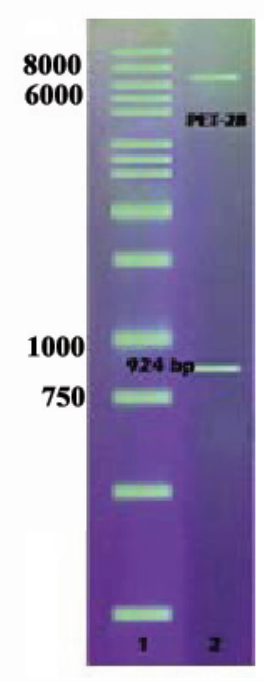

Figure 2. Representative agarose gel for demonstrating the oipA-pET28a construct after digestion by BamHI and XhoI. Lane 1: DNA marker; Lane 2: oipA (924 bp) and pET-28a after digestion (5369 bp)

Iranian isolate was compared with 25 oipA DNA sequences $\left(\mathrm{E}_{\mathrm{value}}=0\right)$. Identity of Iranian oipA genes with other published oipA genes in GeneBank was in the ranges $92-96 \%$ (Table 2, Figure 5). Identity of oip $A$ gene obtained in the present work with that of JHP0581 oipA gene from China (AF479754), was $94 \%$ (5). Its identity with the oipA from Japan and USA was $92-94 \%$ (AF233660-AF233683), respectively (4) and with that of the Mexican (FJ899816FJ899835) was 93-96\% (6). Also, its identity with
$\mathbf{A}$

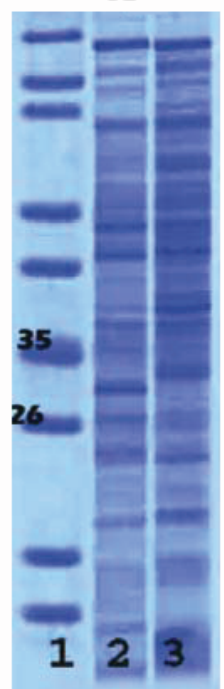

B

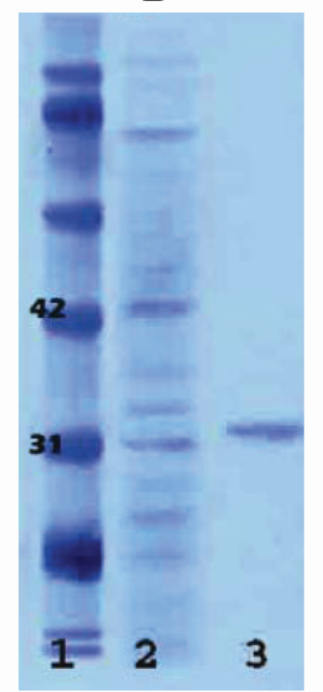

C

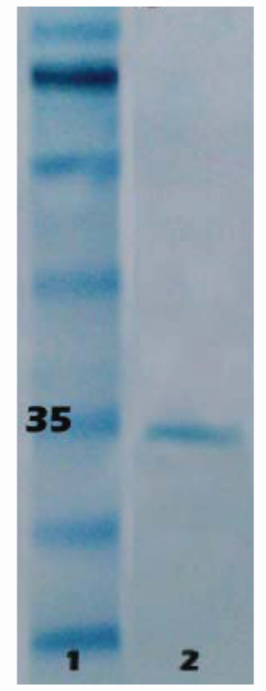

Figure 3. Protein expression in E. coli $\mathrm{BL}$ (DE3) on SDSPAGE gel. A: Lane 1-MW marker, lane 2-control (-), lane 3Recombinant clone. (Coomassie stained); B: Purified recombinant protein: Lane 1- MW marker, lane 2-control (), lane 3-recombinant clone. (Coomassie stained). C: Western Blotting with anti-His-tag monoclonal antibody: Lane 1-MW marker, lane 2-Recombinant clone

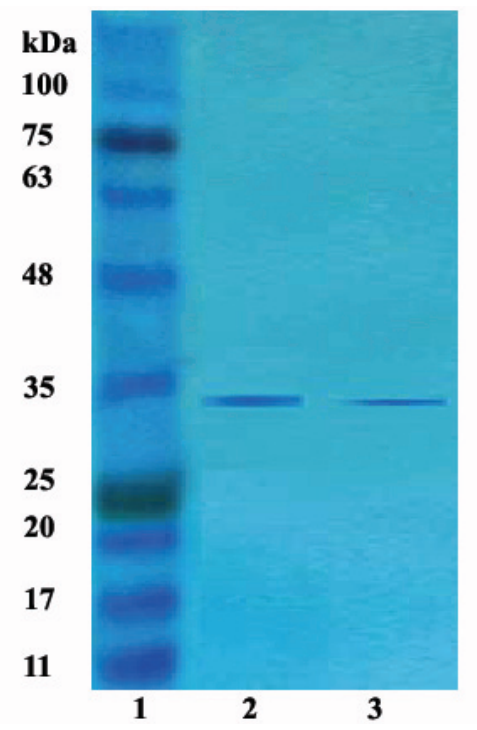

Figure 4. Western blot analysis of OipA recombinant clone with anti-OipA specific antibodies. Lane 1-prestained protein MW marker, lane 2- recombinant OipA clone, lane 3OipA from strain S15 (strain used for cloning)

oipA from $H$. pylori $\mathrm{B} 8 H$. pylori strain and standard $26695 H$. pylori strain were $94 \%$ and $95 \%$, respectively. The CT repeat of the Iranian oipA gene had five CT repeat at the $5^{\prime}$ end of the strand.

\section{Discussion}

To obtain a protective vaccine against $H$. pylori infection, numerous investigators have evaluated antigenic properties of $H$. pylori virulence factors including urease, catalase, VacA, CagA, NapA, GroES, AlpA, BabA, HpaA and SOD recombinant proteins (11-16). Among them, OipA may be one of the most important as it is present in $97.5 \%$ and $70 \%$ of patients with peptic ulcer and chronic gastritis, respectively (4). Furthermore, it was demonstrated that expression of oipA by $H$. pylori, enhances the level of IL-8 in serum by increasing the neutrophil infiltration in the gastric mucosa (17). However, there are many unanswered questions about its invivo expression status, polymorphism and regulation. It was suggested that the isolates containing the cag pathogenicity island ( $\operatorname{cag} A$ positive status), typically also have oip $A$ with functional status "on", probably for their inter-related regulation (18). For this purpose, in the present work, a strain with $\operatorname{cag} A$ positive status was selected for molecular cloning (Figure 1). Furthermore, production of a functional OipA protein requires intact signal region of the gene and the 
absence of any mutation downstream of the gene. For this purpose and for molecular cloning, we selected a strain expressing well the 33-34 kDa OMP. In addition, to evaluate the association of oip $A$ status with $H$. pylori related clinical symptoms; we used a local clinical strain isolated from patients with severe clinical symptoms. Successful cloning of entire oipA gene able to express the $33-34 \mathrm{kDa}$ protein would explain that this is the first work obtaining a functional oipA clone in Iran (Figures 2-4). By comparison of DNA sequence of this Iranian clone with the sequences of other oip $A$ genes cluster, high grade similarity was observed with Mexican oip $A$ and with that of Chinese oipA to a lesser extent (Table 2, Figure 5). The oipA polymorphism, which is well documented by Torres-Morquecho et al. (2010), has demonstrated a possible relationship to the severity of H. pylori-related symptoms and outcomes. This relationship is observed more in the isolates from south regions (6). The fact that the strain used in this work has been isolated from a patient with more severe symptoms, association of this 33-34 kDa protein with more severe outcome of $H$. pylori infection in Iranian population may also be suggested. Furthermore, presence of more identity between oip $A$ of this work and that of Mexican, would explain its similar role in the risk for gastroduodenal diseases among different human populations. However, comparison of complete oipA gene sequences from multiple Iranian patients may better clarify this suggestion.

\section{Acknowledgements}

The Vice Chancellor for Research, Alzahra University, Tehran, Iran, have supported this work. Special thanks for Prof. Jozef Anné from Rega Institute for Medical Research, KU Leuven, Minderbroedersstraat 10 blok x-bus 1030, B-3000 Leuven, Belgium for valuable comments on the manuscript.

\section{Conflict of interest}

The authors have no substantial financial or commer-

Table 2. The blasting of the oipA gene from Iranian isolates with oipA from Gene Bank

\begin{tabular}{|c|c|c|c|c|c|}
\hline Description of clone gene & Query cover & Identity & Accession & Reference & bp \\
\hline clone 259f5oipA & $87 \%$ & $95 \%$ & FJ869826 & (6) & 860 \\
\hline clone 24a3oipA & $88 \%$ & $95 \%$ & FJ869815 & (6) & 863 \\
\hline clone 252a2oipA & $87 \%$ & $95 \%$ & FJ869823 & (6) & 861 \\
\hline clone 44c4oipA & $87 \%$ & $95 \%$ & FJ869818 & (6) & 857 \\
\hline strain SS1oipA & $89 \%$ & $94 \%$ & AF233683 & (4) & 896 \\
\hline clone 254a10oipA & $88 \%$ & $94 \%$ & FJ869824 & (6) & 881 \\
\hline clone 44c2oipA & $87 \%$ & $94 \%$ & FJ869817 & (6) & 855 \\
\hline clone 259a1oipA & $87 \%$ & $94 \%$ & FJ869835 & (6) & 866 \\
\hline strain GI2060oipA & $89 \%$ & $94 \%$ & AF233682 & (4) & 894 \\
\hline clone 69a2oipA & $87 \%$ & $94 \%$ & FJ869819 & (6) & 855 \\
\hline clone JHP0581oipA & $89 \%$ & $94 \%$ & AF479754 & (5) & 924 \\
\hline clone 309a11oipA & $87 \%$ & $94 \%$ & FJ869830 & (6) & 849 \\
\hline clone 236c6oipA & $87 \%$ & $94 \%$ & FJ869820 & (6) & 853 \\
\hline clone 33a1oipA & $85 \%$ & $94 \%$ & FJ869816 & (6) & 824 \\
\hline clone 251i4oipA & $83 \%$ & $94 \%$ & FJ869822 & (6) & 808 \\
\hline clone 256c2oipA & $83 \%$ & $94 \%$ & FJ869825 & (6) & 809 \\
\hline clone 261a1oipA & $87 \%$ & $93 \%$ & FJ869827 & (6) & 853 \\
\hline strain JK35oipA & $87 \%$ & $92 \%$ & AF233662 & (4) & 886 \\
\hline strain JK51oipA & $89 \%$ & $92 \%$ & AF233667 & (4) & 893 \\
\hline clone 307c4oipA & $87 \%$ & $96 \%$ & FJ869829 & (6) & 897 \\
\hline clone 550c12oipA & $47 \%$ & $95 \%$ & FJ869832 & (6) & 459 \\
\hline clone 248f9oipA & $46 \%$ & $95 \%$ & FJ869821 & (6) & 453 \\
\hline clone 475a3oipA & $46 \%$ & $95 \%$ & FJ869831 & (6) & 456 \\
\hline clone 291c11oipA & $46 \%$ & $94 \%$ & FJ869828 & (6) & 456 \\
\hline clone 249a5oipA & $46 \%$ & $94 \%$ & FJ869834 & (6) & 448 \\
\hline
\end{tabular}




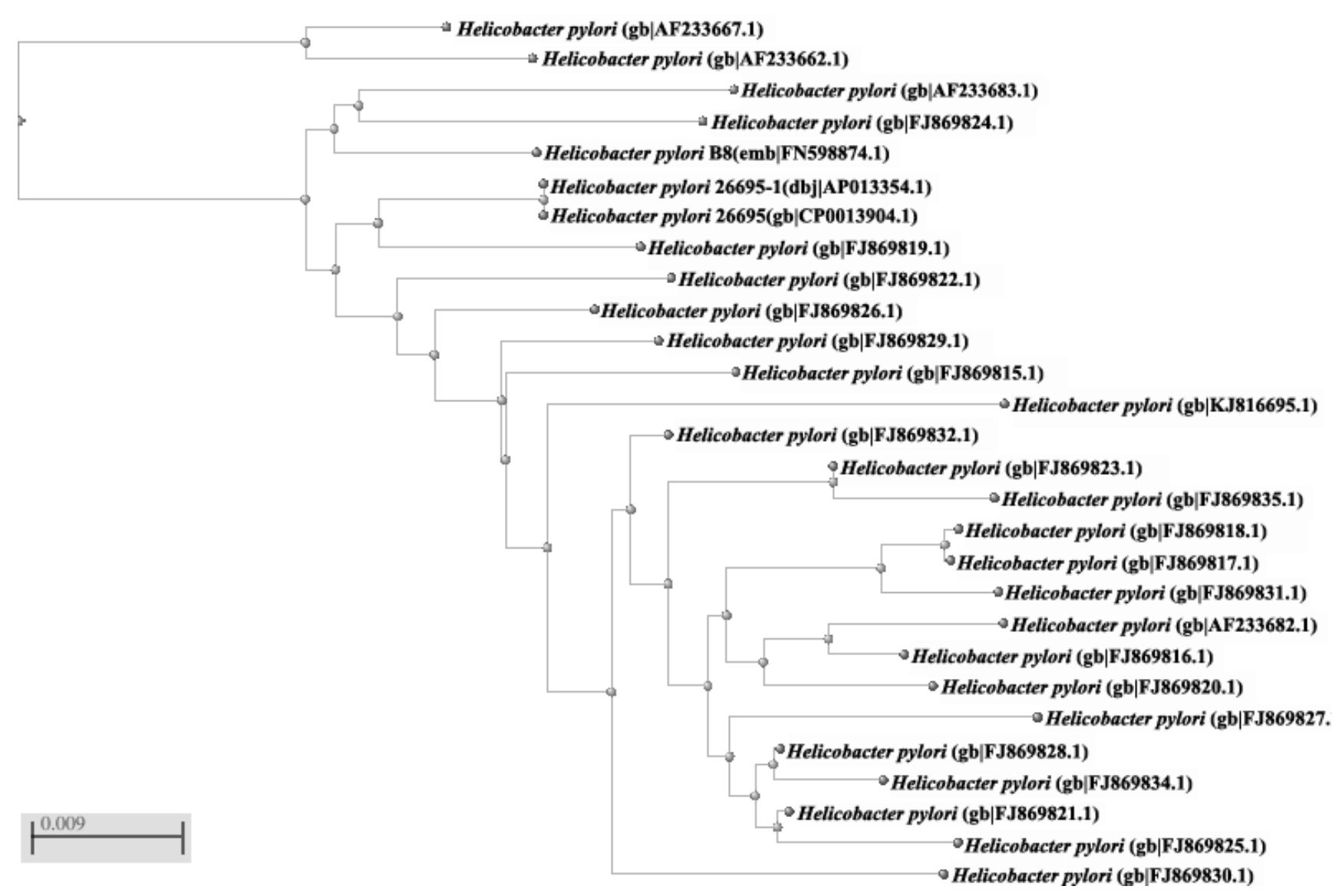

Figure 5. Phylogenic tree of the oipA gene from Iranian isolates (S15) with oipA from Gene Bank

cial conflicts of interest with the current work or its publication.

\section{Authors' Contributions}

Mahboubi $\mathrm{M}$ and Falsafi $\mathrm{T}$, designed the study and wrote the manuscript, also provided vital analytical tools; Mahboubi M performed the majority of experiments; Sadeghizadeh M, participated to analytical tools and critically reading of the manuscript.

\section{References}

1. Sgouros SN, Bergele C. Clinical outcome of patients with Helicobacter pylori infection: the bug, the host, or the environment? Postgrad Med J. 2006;82(967):338342.doi:10.1136/pgmj.2005.038273

2. Tabassam FH, Graham DY, Yamaoka Y. OipA plays a role in Helicobacter pylori-induced focal adhesion kinase activation and cytoskeletal re-organization. Cell Microbiol. 2008;10(4):10081020. doi:10.1111/j.1462-5822.2007.01104.x

3. Yamaoka Y, Kodama T, Graham DY, Kashima K. Search for putative virulence factors of Helicobacter pylori: the lowmolecular-weight $(33-35 \mathrm{~K})$ antigen. Dig Dis Sci. 1998;43(7):1482-1487.doi:10.1023/A:1018850412148
4. Yamaoka Y, Kwon DH, Graham DY. A M(r) 34,000 proinflammatory outer membrane protein (oipA) of Helicobacter pylori. Proc Natl Acad Sci U S A. 2000;97(13):75337538.doi:10.1073/pnas.130079797

5. Chen DR, Huang AL, Tao XH, Wang PL, Jiang Z. Cloning and sequence analysis of gene oipA encoding an outer membrane protein of human Helicobacter pylori. World $J$ Gastroenterol. 2004;10(21):3205-3207.

6. Torres-Morquecho A, Giono-Cerezo S, Camorlinga-Ponce M, Vargas-Mendoza CF, Torres J. Evolution of bacterial genes: evidences of positive Darwinian selection and fixation of base substitutions in virulence genes of Helicobacter pylori. Infect Genet Evol. 2010;10(6):764-776.doi:10.1016/j.meegid.2010.04.005

7. Teymournejad O, Mobarez AM, Hassan ZM, Noori S, Moazzeni SM, Khoramabadi N. Cloning, Expression, Purification and Toxicity Evaluation of Helicobacter pylori Outer Inflammatory Protein A. Ind $J$ Microbiol. 2013;53(4):391-394.doi:10.1007/s12088-013-0383-2

8. Falsafi T. Association between efficient adhesion to HEp-2 cells and severity of gastritis in Helicobacter pylori strains isolated from children. Iranian Journal of Microbiology. 2009;1(1):23-30.doi:10.1186/1471-230X-2-20

9. Faundez G, Troncoso M, Figueroa G. cagA and vacA in strains of Helicobacter pylori from ulcer and non-ulcerative dyspepsia 
patients. BMC Gastroenterol. 2002;2(20):10.doi:10.1186/1471230X-2-20

10. Landarani Z, Falsafi T, Mahboubi M, Behzad Lameh-rad B. Immunological detection of $34 \mathrm{KDa}$ outer membrane protein as a functional form of OipA in clinical isolates of Helicobacter pylori. Iran J Microbiol. 2014;6(5):324-329.

11. Every AL, Stent A, Moloney MB, Ng GZ, Skene CD, Edwards SJ, Sutton P. Evaluation of superoxide dismutase from Helicobacter pylori as a protective vaccine antigen. Vaccine. 2011;29(7):1514-1518.doi:10.1016/j.vaccine.2010.12.019

12. Ferrero RL, Thiberge JM, Kansau I, Wuscher N, Huerre M, Labigne A. The GroES homolog of Helicobacter pylori confers protective immunity against mucosal infection in mice. Proc Natl Acad Sci USA. 1995;92(14):6499-6503.doi:10.1073/pnas.92.14.6499

13. O'Riordan AA, Morales VA, Mulligan L, Faheem N, Windle HJ, Kelleher DP. Alkyl hydroperoxide reductase: a candidate Helicobacter pylori vaccine. Vaccine. 2012;30(26):38763884.doi:10.1016/j.vaccine.2012.04.002

14. Radcliff FJ, Hazell SL, Kolesnikow T, Doidge C, Lee A. Catalase, a novel antigen for Helicobacter pylori vaccination. Infect Immun.
1997;65(11):4668-4674.doi:10.1128/CDLI.9.4.784-788.2002

15. Satin B, Del Giudice G, Della Bianca V, Dusi S, Laudanna C, Tonello F, Kelleher D, Rappuoli R, Montecucco C, Rossi F. The neutrophil-activating protein (HP-NAP) of Helicobacter pylori is a protective antigen and a major virulence factor. $J$ Exp Med. 2000;191(9):1467-1476.doi:10.1111/j.1574-695X.2007.00258.x

16. Xue J, Bai Y, Chen Y, Wang JD, Zhang ZS, Zhang YL, Zhou DY. Expression of Helicobacter pylori AlpA protein and its immunogenicity. World J Gastroenterol. 2005;11(15):22602263.doi:10.3748/wjg.v11.i15.2260

17. Yamaoka Y, Kikuchi S, el-Zimaity HM, Gutierrez O, Osato MS, Graham DY. Importance of Helicobacter pylori oipA in clinical presentation, gastric inflammation, and mucosal interleukin 8 production. Gastroenterol. 2002;123(2):414424.doi:10.1053/gast.2002.34781

18. Yamaoka Y, Ojo O, Fujimoto S, Odenbreit S, Haas R, Gutierrez O, El-Zimaity HM, Reddy R, Arnqvist A, Graham DY. Helicobacter pylori outer membrane proteins and gastroduodenal disease. Gut. 2006;55(6):775-7781.doi:10.1136/gut.2005.083014 